\title{
Association Between Fecal Calprotectin Levels and \\ Small-bowel Inflammation Score in Capsule Endoscopy : A \\ Multicenter Retrospective Study
}

\section{Koulaouzidis, Anastasios}

2016-07

Koulaouzidis , A , Sipponen , T , Nemeth , A, Makins , R , Kopylov , U , Nadler , M , Giannakou, A, Yung, D E, Johansson, G W , Bartzis , L, Thorlacius , H, Seidman , E G , Eliakim , R , Plevris , J N \& Toth , E 2016, ' Association Between Fecal Calprotectin Levels and Small-bowel Inflammation Score in Capsule Endoscopy : A Multicenter Retrospective Study ' , Digestive Diseases and Sciences , vol. 61 , no. 7 , pp. 2033-2040 . https://doi.org/10.1007/s10620-016-410

http://hdl.handle.net/10138/224099

https://doi.org/10.1007/s10620-016-4104-7

publishedVersion

Downloaded from Helda, University of Helsinki institutional repository.

This is an electronic reprint of the original article.

This reprint may differ from the original in pagination and typographic detail.

Please cite the original version. 


\title{
Association Between Fecal Calprotectin Levels and Small-bowel Inflammation Score in Capsule Endoscopy: A Multicenter Retrospective Study
}

\author{
Anastasios Koulaouzidis ${ }^{1}$ - Taina Sipponen ${ }^{2} \cdot$ Artur Nemeth $^{3} \cdot$ Richard Makins $^{4}$. \\ Uri Kopylov $^{5}$ - Moshe Nadler ${ }^{6}$ - Andry Giannakou ${ }^{7}$ - Diana E. Yung ${ }^{1}$. \\ Gabriele Wurm Johansson ${ }^{3}$ - Leonidas Bartzis ${ }^{1}$ - Henrik Thorlacius ${ }^{8}$.

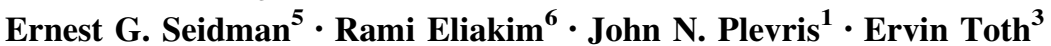

Received: 2 November 2015/ Accepted: 28 February 2016/Published online: 23 March 2016

(c) Springer Science+Business Media New York 2016

\begin{abstract}
Background Accurate inflammation reporting in capsule endoscopy (CE) is important for diagnosis and monitoring of treatment of inflammatory bowel disease (IBD). Fecal calprotectin (FC) is a highly specific biomarker of gut inflammation. Lewis score (LS) was developed to standardize quantification of inflammation in small-bowel (SB) CE images.

Goals Multicenter retrospective study aiming to investigate correlation between LS and FC in a large group of patients undergoing $\mathrm{CE}$ for suspected or known smallbowel IBD, and to develop a model for prediction of $\mathrm{CE}$ results (LS) based on FC levels.
\end{abstract}

Diana E. Yung

diana.e.yung@gmail.com

1 Endoscopy Unit, Centre for Liver and Digestive Disorders, The Royal Infirmary of Edinburgh, 51 Little France Crescent, Edinburgh, UK

2 Department of Gastroenterology, University of Helsinki and Helsinki University Hospital, Helsinki, Finland

3 Department of Gastroenterology, Skåne University Hospital, Lund University, Malmö, Sweden

4 Department of Gastroenterology, Gloucestershire Hospitals NHS Foundation Trust, Cheltenham, UK

5 Division of Gastroenterology, McGill University Health Center, Montreal, QC, Canada

6 Department of Gastroenterology, Sheba Medical Center, Sackler School of Medicine, Tel-Aviv University, Tel-Aviv, Israel

7 Faculty of Economics and Management, Open University of Cyprus, Nicosia, Cyprus

8 Department of Surgery, Skåne University Hospital, Lund University, Malmö, Sweden
Study Five academic centers and a district general hospital offering CE in UK, Finland, Sweden, Canada, and Israel. In total, 333 patients were recruited. They had small-bowel CE and FC done within 3 months.

Results Overall, correlation between FC and LS was weak $\left(r_{\mathrm{s}}: 0.232, P<0.001\right)$. When two clinically significant FC thresholds (100 and $250 \mu \mathrm{g} / \mathrm{g}$ ) were examined, the $r_{\mathrm{s}}$ between FC and LS was 0.247 (weak) and 0.337 (moderate), respectively $(P=0.307)$. For clinically significant $(\mathrm{LS} \geq 135)$ or negative $(\mathrm{LS}<135)$ for $\mathrm{SB}$ inflammation, ROC curves gave an optimum cutoff point of FC $76 \mu \mathrm{g} / \mathrm{g}$ with sensitivity 0.59 and specificity 0.41 . Limitations: Retrospective design.

Conclusions LS appears to show low correlation with FC as well as other serology markers of inflammation. FC does not appear to be a reliable biomarker for significant smallbowel inflammation. Nevertheless, FC level $\geq 76 \mu \mathrm{g} / \mathrm{g}$ may be associated with appreciable visual inflammation on small-bowel CE in patients with negative prior diagnostic workup.

Keywords Capsule endoscopy - Fecal calprotectin · Lewis score . Small-bowel inflammation · Monocyte count $\cdot$ C-reactive protein · Multicenter study

\section{Introduction}

Capsule endoscopy (CE) is the prime modality for accurate, non-invasive, and pain-free investigation of the small bowel [1]. In order to standardize reporting of smallbowel inflammation using $\mathrm{CE}$, two scoring indices have been developed: the Lewis score (LS) and the Capsule Endoscopy Crohn's Disease Activity Index (CECDAI) [24]. Both scores are based on parameters and descriptors of 
inflammatory change and have been externally validated in several reports [5-8]. However, they are of limited discriminatory ability, and it is still unclear how accurately they measure the degree of mucosal inflammation $[6,9]$.

Calprotectin was first isolated from human granulocyte cells by Fagerhol et al. [10]. Calprotectin is a major component of the cytosol of neutrophils and, to a lesser extent, monocytes and activated macrophages, released in feces upon leukocyte and epithelial activation [11-13]. In the presence of calcium, calprotectin is resistant to degradation and stable in feces at room temperature for up to 7 days $[11,14]$. Fecal calprotectin (FC) 'leaks' into the gut lumen through inflamed mucosa therefore reflecting the amount of leukocyte cell activation, migration, and death [15]. Although FC is not disease specific, a recent meta-analysis showed an excellent correlation of FC with the severity of mucosal inflammation. At a cutoff level of $100 \mu \mathrm{g} / \mathrm{g}$, FC can distinguish inflammatory bowel disease (IBD) from non-inflammatory conditions [16]. Therefore, many experts consider FC a reliable and highly specific biomarker of inflammation [9, 11]. There are conflicting reports suggesting that the correlation between FC and mucosal inflammation may be weaker in small-bowel inflammation in comparison with the colon. Monoclonal, polyclonal, and combination ELISA (quantitative), and bedside immune-chromatographic (semiquantitative) methods have been developed (and validated) for FC measurement [12].

Recently, we showed that measurement of FC levels prior to referral for $\mathrm{CE}$ is a useful tool to select patients with possible small-bowel IBD [17]. In this single-center study, FC $>100 \mu \mathrm{g} / \mathrm{g}$ is good predictor of positive smallbowel CE findings, while $\mathrm{FC}>200 \mu \mathrm{g} / \mathrm{g}$ was associated with higher $\mathrm{CE}$ diagnostic yield $(65 \%)$ and confirmed small-bowel inflammation in $50 \%$ of cases. Hence, it is reasonable to consider that strong correlation should exist between FC levels and LS [7-9]. However, in a separate cohort of patients with suspected, isolated small-bowel disease, LS showed strong correlation with FC at levels $<100 \mu \mathrm{g} / \mathrm{g}$ [8]. The overall correlation between FC and LS is moderate at best [18]. This is certainly consistent with the high-negative predictive value (NPV) of FC [9]. Nonetheless, in individuals with higher FC levels, LS does not correlate well, and this can have impact on both patient selection for $\mathrm{CE}$ as well as with final outcomes.

The primary aim of this multicenter, retrospective study was to investigate the correlation between LS and FC in a larger group of patients who underwent $\mathrm{CE}$ for suspected or known small-bowel IBD.

Our secondary aim was to develop a model for prediction of CE results (LS) based on FC levels.

\section{Materials and Methods}

\section{Patients and CE Procedure}

This was a retrospective, multicenter study. The study cohort included all consecutive patients who underwent small-bowel CE in five academic referral centers (UK, Finland, Sweden, Canada, and Israel) and a large district general hospital (UK), from January 2010 to December 2013, with clinical suspicion of IBD or for IBD reassessment. Patients having normal ileocolonoscopy, without histological confirmation of Crohn's Disease (CD) on any biopsy material examined, were also eligible. A FC measurement within 3 months from the time of CE was considered necessary for inclusion. The absence of a bidirectional digestive endoscopy in the preceding period (up to a year before CE) was considered an exclusion criterion. Other causes of raised CRP or monocytes were excluded following review of patient case notes. Clinical and demographic data on age, gender, and CE indications were extracted from the patients' files and/or electronic hospital records. A small part of the UK and Swedish data may have been used in a previous publication [25].

The CE was performed with PillCam ${ }^{\circledR}$ SB2/SB3 (Given ${ }^{\circledR}$ Imaging Ltd, Yokneam, Israel) and MiroCam ${ }^{\circledR}$ (IntroMedic Co, Seoul, South Korea), according to local hospital protocols. Technical characteristics of these systems can be found elsewhere in the literature $[19,20]$. Bowel preparation, where used, was polyethylene glycol (PEG) 2 or 4 lt. Prokinetics, where used, was in the form of domperidone (5-10 mg orally) and/or metoclopramide (10 mg intramuscularly) [21].

\section{Fecal Calprotectin, C-Reactive Protein, and Monocyte Count}

FC was measured with monoclonal/polyclonal ELISA (CALPRO AS, Lysaker, Norway; reference range 0-50 $\mu \mathrm{g} / \mathrm{g}$ ) or immune-chromatographic assay (Buhlmann's Quantum Blue, Basel, Switzerland; reference range: normal $<50 \mu \mathrm{g} /$ g; "gray zone" 51-99 $\mu \mathrm{g} / \mathrm{g}$; positive $>100 \mu \mathrm{g} / \mathrm{g}$ ) [11]. For the purpose of further statistical analysis, where FC $<20 \mu \mathrm{g} /$ $\mathrm{g}$, i.e., undetectable, the value 0 was used; for the semiquantitative assays, for values $>300 \mu \mathrm{g} / \mathrm{g}$, the $300 \mu \mathrm{g} / \mathrm{g}$ was used. The C-reactive protein (CRP) and monocyte count were normal across sites if levels were $<5$ and $<0.8 \mathrm{ng} / \mathrm{l}$, respectively.

\section{Lewis Score Calculation}

All videos were reviewed by experienced $\mathrm{CE}$ readers (AK, TS, AN, ET, RM, GW, ES and RE). LS was calculated using the integrated LS Calculator $\left(R A P I D^{\circledR}\right.$, Given ${ }^{\circledR}$ Imaging Ltd, Yokneam, Israel) under white light or blue mode review [22]; where the calculator was not available 
(MiroView ${ }^{\circledR}$, IntroMedic Co, Seoul, South Korea), the calculation was performed manually. LS is based on the number and distribution of intestinal segments with villous edema, ulceration, and stenosis. To calculate the LS, the small bowel is first divided into equal transit thirds (tertiles). The final LS represents the highest tertile or the score with stenosis, if demonstrated [23]. Eventually, the LS allows small-bowel inflammatory activity to be classified into three grades: (1) normal or clinically insignificant mucosal inflammatory change (LS < 135); (2) mild disease $(135 \leq \mathrm{LS}<790)$; and (3) moderate-to-severe disease (LS $\geq 790$ ) $[2,5,6]$. The CE date, FC measurement date, and time difference in days between the two was also calculated [8].

\section{Statistical Analysis}

Baseline quantitative data are presented as median and inter-quartiles range (IQR). For nominal variables, the Chi-square test or Fisher's exact test were used as appropriate. Student's $t$ test was used for quantitative variables with normal distribution. Spearman's rank correlation coefficient (rho; $r_{\mathrm{s}}$ ) was used to assess the correlation between LS and FC. The strength of correlation was defined as follows: $r_{\mathrm{s}}$ values $\leq 0.1$ were considered to denote no correlation; $0.1-0.3$ weak to modest; $0.3-0.49$ moderate; $0.5-0.79$ strong; and, $\geq 0.8$ very strong correlation [24].

In order to detect the association between FS and LS adjusted for other factors, a multivariate linear regression analysis was used. The initial model contained age and monocyte count as adjustment factors of time lag between FC measurement and small-bowel CE. The model was subjected to a backwards elimination procedure using a multivariate linear regression analysis using the likelihood ratio test. A two-tailed probability $(P)$ value $<0.05$ was considered to be statistically significant. In addition, a receiver operating characteristic (ROC) analysis was conducted in order to determine the optimum cutoff point of FC results using the dichotomization of LS as explained in the previous paragraph. Statistical analyses were carried out in $\mathrm{R}$ statistical package.

\section{Ethics Consideration}

This study was conducted in accordance with local research ethics guidelines. After review by the local ethics committee(s), further specific ethical review and approval was not required, as the study was considered a service evaluation/clinical audit based on previously collected clinical data, with no additional patient intervention, obtained as part of regular clinical care.

\section{Results}

\section{Patients and Capsule Endoscopy Data}

In the aforementioned period, 333 (119M/214F; median age: 41 years; IQR: 25) patients who fulfilled the study inclusion criteria were referred for $\mathrm{CE}$ due to clinical suspicion of small-bowel IBD $(n=287 ; 98 \mathrm{M} / 189 \mathrm{~F}$; median age: 41 years; IQR: 26) or suspicion of small-bowel inflammation reactivation in patients with known $\mathrm{CD}(n=46 ; 21 \mathrm{M} / 25 \mathrm{~F}$; median age: 34.5 years; IQR: 24). Two different small-bowel CE systems were used (PillCam ${ }^{\circledR} \mathrm{SB}: 150 / \mathrm{MiroCam}^{\circledR}$ : 183); in three patients the capsule endoscope $\left(2\right.$ PillCamSB $^{\circledR}$, 1 MiroCam $^{\circledR}$ ) was retained in the stomach for the entire period of the recording, hence no LS data were available. These cases were excluded from further analysis. Symptoms were mainly diarrhea, anemia, weight loss, and/or abdominal pain, Table 1.

\section{Fecal Calprotectin}

\section{Clinically Important FC Thresholds}

FC measurements were performed with a quantitative ELISA in 280 patients and with semiquantitative assays in the remainder $(n=50)$. Overall, for the entire dataset $(n=330)$, correlation between FC and LS was weak $\left(r_{\mathrm{s}}\right.$ : $0.232, P<0.001)$. When the two clinically significant FC thresholds of 100 and $250 \mu \mathrm{g} / \mathrm{g}$ were examined [11, 17], irrespective of the FC assay used, the $r_{\mathrm{s}}$ between FC and LS for the two threshold levels was 0.247 (weak) and 0.337 (moderate), respectively $(P=0.307)$. The median values (with range; IQR) for $\mathrm{FC}, \mathrm{LS}$ and the time interval between FC measurement and small-bowel CE were $90(15,255$; $240) \mu \mathrm{g} / \mathrm{g}$, and $0(0,337.5 ; 337.5)$ and $0(0,62.75 ; 62.75)$ days, respectively. Furthermore, no LS/FC correlation difference was recorded between the two small-bowel $\mathrm{CE}$ systems, $(P=0.118)$.

In the quantitative FC (ELISA) subgroup $(n=280)$, the correlation between FC and LS was moderate $\left(r_{\mathrm{s}}: 0.385, P\right.$ : 0.0 ), as previously shown $[8,25]$. The median values (with range; IQR) for $\mathrm{FC}, \mathrm{LS}$, and the time interval between $\mathrm{FC}$ measurement and small-bowel CE were $28 \mu \mathrm{g} / \mathrm{g}(9,220 ; 211)$, and $0(0,339.75 ; 339.75)$ and 14.5 days $(0,46.75 ; 46.75)$, respectively. In this subgroup, $150 \mathrm{CE}$ were performed with MiroCam ${ }^{\circledR}$ and the remainder $(n=130)$ with PillCam ${ }^{\circledR}$ SB. No statistical difference between FC levels (100.37 \pm 191.24 vs $90.71 \mu \mathrm{g} / \mathrm{g} ; P=0.649$ ), time interval between $\mathrm{FC} / \mathrm{CE}$ (28.4 \pm 39.4 vs $20.63 \pm 29.5$ days; $P=0.059$ ), prokinetic use $(P=0.547)$, or bowel prep use $(P=0.717)$ between the two CE subgroups was noted, Table $2 \mathrm{a}, \mathrm{b}$. 
Table 1 Indications for referral for $\mathrm{CE}$

\begin{tabular}{lc}
\hline Indication & Number of patients (\% of total) \\
\hline Diarrhea & $112(33.6)$ \\
Abdominal pain & $104(31.2)$ \\
Iron deficiency anemia & $62(18.6)$ \\
Raised FC & $26(7.8)$ \\
Weight loss & $23(6.9)$ \\
OGIB & $19(5.7)$ \\
Abnormal radiological investigations & $11(3.3)$ \\
Background of celiac disease, autoimmune disease or IBD & $11(3.3)$ \\
Nutritional deficiencies/malabsorption, e.g., B12/folate, albumin & $9(2.7)$ \\
Family history of IBD & $6(1.8)$ \\
Perianal fistula & $6(1.8)$
\end{tabular}

Please note that numbers do not add up to study size of 333 as many patients had more than one indication for referral

$F C$ fecal calprotectin, $I B D$ inflammatory bowel disease

(a) Comparison of subgroups

\begin{tabular}{lll}
\hline & Quantitative FC & Semiquantitative FC \\
\hline $\mathrm{N}$ & 280 & 50 \\
Median FC $(\mu \mathrm{g} / \mathrm{g})($ range IQR) & $28(9-220 ; 211)$ & $145(105.75-300 ; 194.25)$ \\
Median LS (range IQR) & $0(0-339.75 ; 339.75)$ & $135(0-287 ; 287)$ \\
Median time from FC to CE (days) (range IQR) & $14.5(0-46.75 ; 46.75)$ & $25(0-474 ; 474)$ \\
\hline
\end{tabular}

(b) Comparison of MiroCam ${ }^{\circledR}$ vs. PillCam ${ }^{\circledR}$ SB2 subgroups in the quantitative FC group

\begin{tabular}{llll}
\hline & MiroCam $^{\circledR}$ & PillCam $^{\circledR}$ SB2 & $P$ value \\
\hline $\mathrm{N}$ & 150 & 130 & \\
Median FC $(\mu \mathrm{g} / \mathrm{g}, \mathrm{SD})$ & $100.37 \pm 191.24$ & $90.71 \pm 166.1$ & 0.547 \\
Time from FC to SBCE (days, SD) & $28.4 \pm 39.4$ & $20.63 \pm 29.5$ & 0.059 \\
Prokinetic use & 55 & 42 & 0.547 \\
Bowel prep used & 54 & 42 & 0.717 \\
\hline
\end{tabular}

$F C$ fecal calprotectin, $I Q R$ inter-quartile range, $L S$ Lewis score, $S D$ standard deviation, $S B C E$ small-bowel capsule endoscopy
In the subgroup of semiquantitative FC $(n=50)$, there was no correlation between FC and LS $\left(r_{\mathrm{s}}:-0.130, P\right.$ : 0.377). In this subgroup, the median values (with range and IQR) for FC and LS were $145 \mu \mathrm{g} / \mathrm{g}(105.75,300 ; 194.25)$, $135(0,287 ; 287)$, respectively. PillCam ${ }^{\circledR} \mathrm{SB}$ was used in 18 and MiroCam ${ }^{\circledR}$ in 32 patients. Furthermore, the median interval between small-bowel $\mathrm{CE}$ and FC was 25 days $(0-474 ; 474)$ (i.e., not significantly different from the quantitative FC group; $P=0.07$ ).

\section{Monocytes and CRP}

The median (range; IQR) monocyte and CRP counts were $0.535(0.41,0.72 ; 0.31)$ and $7(3,15 ; 12)$, respectively. The correlation between monocyte count and LS was weakly negative $\left(r_{\mathrm{s}}:-0.019, P: 0.732\right)$, while the relevant value for CRP was $r_{\mathrm{s}}:-0.095, P: 0.086$. It has been reported that the $\mathrm{CRP} /$ monocyte ratio represents the acute phase of inflammation [26]. There were 73 complete datasets (ratio, FC and LS) with measurements obtained \pm 7 days around the CE (median: 0 days, IQR: 0 days). The median value of the ratio was $12(5.21,24.47 ; 24.25)$, and the correlation of the ratio with FC and LS was $r_{\mathrm{s}}: 0.14(P: 0.235)$ and $r_{\mathrm{s}}$ : 0.02 ( $P: 0.865)$, respectively.

\section{Model Creation}

In order to investigate the potential association between LS and FC, both variables were log-transformed. The final model for the association of LS and FC was found to be: 


$$
\begin{aligned}
\log (\mathrm{LS}+1)= & -1.05-0.0087 \times \text { time lag simplistic } \\
& +1.0471 \times \log (\mathrm{FC}+1)
\end{aligned}
$$

Other predictors such as age $(P=0.902)$ and monocyte count $(P=0.805)$ were eliminated from the initial model during the backwards elimination procedure. The results of the final model are provided in Table 3, where the intercept $(P=0.269)$ was kept as it was found that the normality of the residuals was violated when this was removed. Furthermore, the model is interpreted as an increase of 1 point in FC gives an increase of 1.0471 in $\log (\mathrm{LS}+1)(95 \%$ CI: $0.679 ; 1.415)$. The latter translates to a 0.389 points increase in LS (95 \% CI: 0.159; 0.832) for a constant FC/ $\mathrm{CE}$ time lag simplicity of zero. Also an increase of 1 point in $\mathrm{FC} / \mathrm{CE}$ time lag gives a decrease of -0.0087 (95\% CI: $-0.016 ;-0.001)$ in $\log (\mathrm{LS}+1)$.

\section{Optimum Cutoff Point of FC}

The analysis using ROC curves gave that the dichotomization of LS at 135 for clinically significant (LS $\geq 135$ ) or negative ( $\mathrm{LS}<135$ ) for SB inflammation gave an optimum cutoff point of FC 76 at $\mu \mathrm{g} / \mathrm{g}$ with sensitivity 0.59 and specificity 0.41 .

\section{Discussion}

FC level in the stool is directly proportional to neutrophils in the intestinal lumen; therefore, its use as biomarker of enteric inflammation and neoplastic lesions has been proposed. One of the main indications for $\mathrm{CE}$ is the direct visualization of the extent, location, and severity of smallbowel inflammation [23]. Others suggest that FC could discriminate between organic and functional intestinal pathology and allow selection of patients who are more likely to benefit from a colonoscopy [16]. Recently, we hypothesized that FC can be used as selection tool for performing $\mathrm{CE}$ in patients with continuing clinical suspicion for small-bowel IBD, despite preliminary negative diagnostic workup [17]. Currently, healthcare systems worldwide are under significant economic strain to provide high-quality care with shrivelling budgets [26, 27]. Therefore, increasing the diagnostic yield of patient workup with inexpensive, accurate, non-invasive investigations, has multiple benefits $[13,28]$.
In the present study, retrospective data on FC, monocyte count, and CRP paired with CE findings (LS was used to quantify small-bowel inflammation in an objective way) [2, 8] were collected from patients with clinical suspicion of small-bowel IBD $(n=287)$, out of which $3 \%$ had ileitis on colonoscopy but inconclusive histology, from high-volume CE centers (UKx2, Finland, Sweden, Canada, Israel). The remainder $(n=43)$ had a history of known $\mathrm{CD}$ and were referred for small-bowel assessment with CE. Experienced $\mathrm{CE}$ reviewers reported the $\mathrm{CE}$ results at each site for the purpose of clinical care/need using white light and/or blue mode (depending on preference per reviewer) [6, 22]. In $84.8 \%$ of cases, FC was measured using a commercially available ELISA (range $0-50 \mu \mathrm{g} / \mathrm{g}$ ). In these patients, CE was performed using the PillCam ${ }^{\circledR} \mathrm{SB}$ in $46.4 \%$ of cases; the remainder was performed with MiroCam ${ }^{\circledR}$. Based on the $\mathrm{CE}$ system used, the two patient subgroups were equivalent in terms of FC levels, time interval between FC measurement and performance of $\mathrm{CE}$, and procedural factors for small-bowel $\mathrm{CE}$ such as the use of a prokinetic and/or a bowel purge (or not). Therefore, we are able to confirm that the lack of an integrated calculator in the MiroCam ${ }^{\circledR}$ proprietary software $\left(\right.$ MiroView ${ }^{\circledR}$ ) notwithstanding the calculated LS had the same correlation with FC levels.

Another finding of this study is low correlation of FC with monocyte count, CRP, CRP/monocyte, and LS, Table 2. The former has been previously shown in studies from our group [15, 25]. Furthermore, elevated CRP, FC, or the combination of both was poorly correlated with detectable small-bowel inflammation [18, 29]. Nevertheless, it is worth noting that when the threshold level of significant SB inflammation, as denoted by LS was shifted from 135 to 350, the correlation of FC and LS was similar at $r_{\mathrm{s}}: 0.07(P: 0.637)$ and $0.09(P: 0.696)$ for the suspected and known $\mathrm{CD}$ group, respectively.

Others have recently confirmed strong inter-observer agreement in determining LS in CE [6]. Höög et al. [7], in a cohort of 30 patients, showed that there was a significant persistent correlation between endoscopic inflammation and FC (at study inclusion and at a year's follow-up). More recently, Olsen et al showed that the proportion of patients with findings on small-bowel CE increased with increasing FC [30]. Nevertheless, in their cohort, a positive FC $(\geq 50 \mathrm{mg} / \mathrm{kg}$ ) had a sensitivity, specificity, positive predictive value (PPV), and negative predictive value (NPV)
Table 3 Model for the association of FC and LS

\begin{tabular}{lclccc}
\hline Model & Coefficients & SE & $t$ value & $\operatorname{Pr}(>|\mathrm{t}|)$ & $95 \%$ CI \\
\hline Intercept & -1.0513 & 0.9466 & -1.11 & 0.269 & $-2.907 ; 0.804$ \\
Time lag FC/CE & -0.0087 & 0.0039 & -2.24 & 0.027 & $-0.016 ;-0.001$ \\
Log $($ FC +1$)$ & 1.0471 & 0.1876 & 5.58 & $<0.001$ & $0.679 ; 1.415$ \\
\hline
\end{tabular}


Fig. 1 Plot of LS in correlation to $\mathrm{FC}$
Plot of LS in correlation to FC

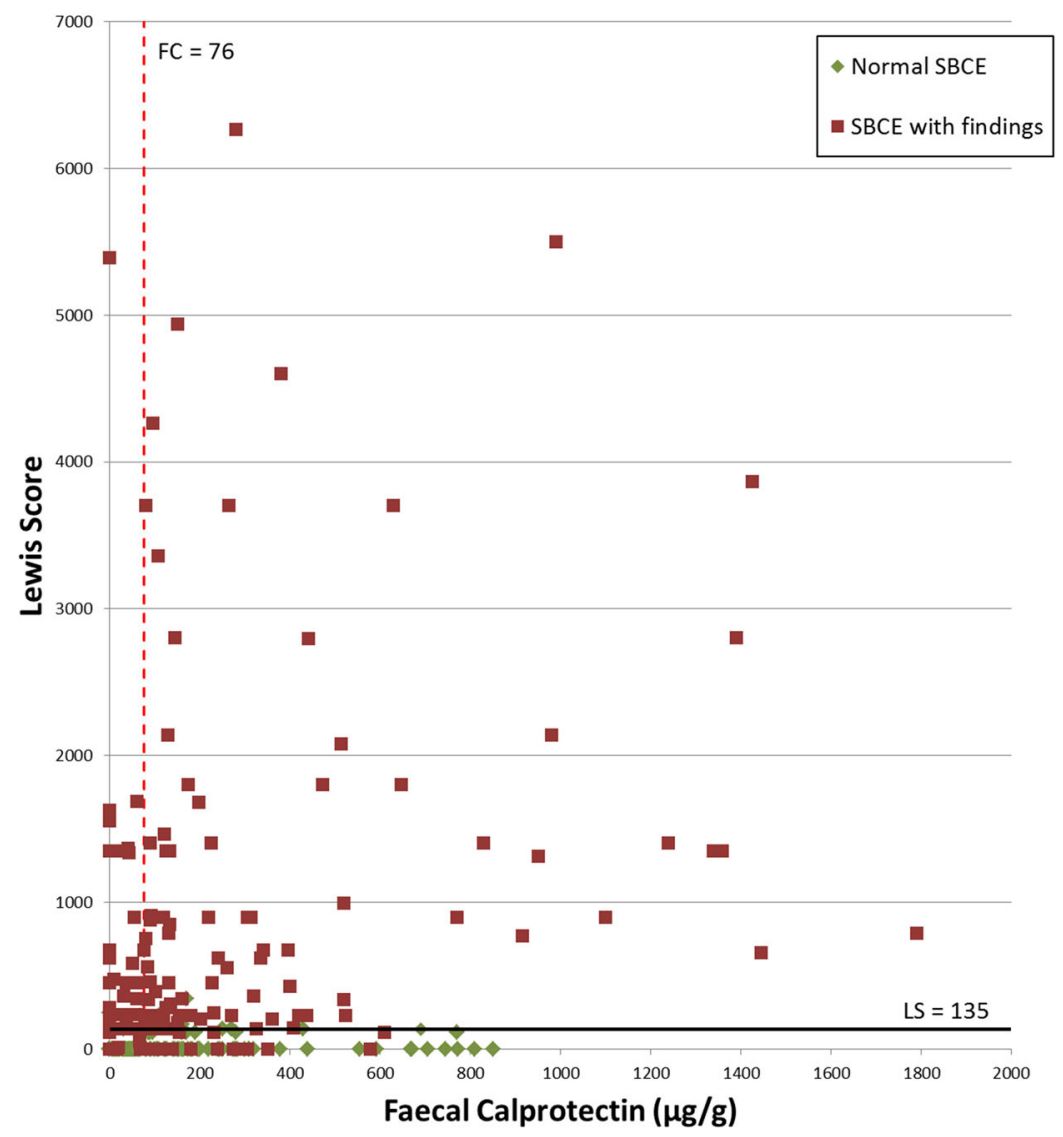

of 54.2, 69.9, 43.3, and $78.2 \%$, respectively. The correlation of FC values with presence of active small-bowel inflammation as detected by magnetic resonance enterography (MRE) was similar to that of CE [29].

Limitations of this study include the lack of formal assessment of the extent of mucosal visualization. As not all patients underwent bowel preparation prior to $\mathrm{CE}$, it is possible that LS could in part be altered by the degree of small-bowel visualization. However, there is a lack of data on LS correlation with the quality of SB visualization. The fact that the CEs in this study were each reviewed by a single reviewer only, despite substantial cumulative experience in $\mathrm{CE}$, could be a further limitation leading to lower diagnostic yield.

This study did not establish a correlation between endoscopic severity, as measured by the LS, and FC or other biomarkers of inflammation. This is likely to reflect deficiencies of the scoring system [25] as well as the study's inherent limitations such as the cutoff level selected. FC may also be a marker of subclinical inflammation; Gisbert and McNicholl [31] found that FC was higher in asymptomatic first-degree relatives of patients with IBD, and FC has been seen to predict relapse in asymptomatic or quiescent CD [32]. Another study has found that FC does not reliably distinguish IBD from malignancy [33], which may-indirectly-suggest that FC is not as good at distinguishing generalized inflammation from foci of inflammation. Furthermore, some studies show FC is a more reliable indicator of colonic than $\mathrm{SB}$ inflammation, i.e., usefulness of FC varies with location of inflammation within the gut, and there is difficulty in establishing correlation due to the heterogeneity of presentations in $\mathrm{CD}$ [34, 35]. Figure 1 shows how LS is generally low in patients with normal SBCE; however these patients have a wide range of FC. Conversely our study also had patients with low FC but high LS, which could have been indicative of a single large lesion, such as an isolated stenosis, yielding a diagnosis. Further prospective studies should be performed to investigate the difference between the equivocal results of our study and other studies which show positive correlation between LS and FC.

Our findings suggest that in patients with strong clinical suspicion of small-bowel $\mathrm{CD}$ and negative bidirectional endoscopy, CE should not be limited to patients with elevated biomarkers only. Especially, CRP and the ratio in particular were not associated with SB inflammation on $\mathrm{CE}$. Moreover, the correlation was moderate for FC, and if this biomarker was used to guide the decision to perform 
$\mathrm{CE}$, at least $40 \%$ of patients will be misdiagnosed. However, the use of single FC measurement per patient for the purpose of this study $[36,37]$, its retrospective nature and the use of different laboratories and FC kits should be considered as additional limitations of this study. Nevertheless, $\mathrm{FC} \geq 76 \mu \mathrm{g} / \mathrm{g}$ may be associated with appreciable inflammation on $\mathrm{CE}$ in patients with negative prior diagnostic workup.

Acknowledgments The authors thank Pirkko Tuukkala and Virpi Pelkonen (both with Helsinki University Central Hospital, Finland) for their invaluable help with the data collection.

Authors' contributions All authors contributed to data collection. Dr. A Koulaouzidis created the first draft. All authors critically reviewed the document and provided changes. All authors approved the final version of the article, including the authorship list.

\section{Compliance with ethical standards}

Conflict of interest Dr. Koulaouzidis received an Given Imaging Ltd/ESGE Ltd research Grant in 2011. He has also accepted material support for research from SynMedUK Ltd. Dr. Seidman has received in-kind research support from Given Imaging/Medtronic Inc., 2011-2015. Rami Eliakim received consultation fees from Given Imaging. The rest of the authors have no disclosure to make.

Competing interests None.

\section{Patient consent None.}

Ethics approval Clinical Audit Department at the Royal Infirmary of Edinburgh.

\section{References}

1. Koulaouzidis A, Iakovidis DK, Karargyris A, Rondonotti E. Wireless endoscopy in 2020: Will it still be a capsule? World $J$ Gastroenterol. 2015;21:5119-5130. doi:10.3748/wjg.v21.i17. 5119.

2. Gralnek IM, Defranchis R, Seidman E, Leighton JA, Legnani P, Lewis BS. Development of a capsule endoscopy scoring index for small bowel mucosal inflammatory change. Aliment Pharmacol Ther. 2008;27:146-154.

3. Gal E, Geller A, Fraser G, Levi Z, Niv Y. Assessment and validation of the new capsule endoscopy Crohn's disease activity index (CECDAI). Dig Dis Sci. 2008;53:1933-1937.

4. Niv Y, Ilani S, Levi Z, et al. Validation of the Capsule Endoscopy Crohn's Disease Activity Index (CECDAI or Niv score): a multicenter prospective study. Endoscopy. 2012;44:21-26. doi:10. 1055/s-0031-1291385.

5. Rosa B, Moreira MJ, Rebelo A, Cotter J. Lewis Score: a useful clinical tool for patients with suspected Crohn's Disease submitted to capsule endoscopy. J Crohns Colitis. 2012;6:692-697. doi:10.1016/j.crohns.2011.12.002.

6. Cotter J. Dias de Castro F, Magalhães J, Moreira MJ, Rosa B. Validation of the Lewis score for the evaluation of small-bowel Crohn's disease activity. Endoscopy. 2015;47:330-335. doi:10. 1055/s-0034-1390894

7. Höög CM, Bark LÅ, Broström O, Sjöqvist U. Capsule endoscopic findings correlate with fecal calprotectin and C-reactive protein in patients with suspected small-bowel Crohn's disease.
Scand J Gastroenterol. 2014;49:1084-1090. doi:10.3109/ 00365521.2014 .920915$.

8. Koulaouzidis A, Douglas S, Plevris JN. Lewis score correlates more closely with fecal calprotectin than Capsule Endoscopy Crohn's Disease Activity Index. Dig Dis Sci. 2012;57:987-993. doi:10.1007/s10620-011-1956-8.

9. Gurudu SR, Leighton JA. Correlation of two capsule endoscopy scoring systems with fecal calprotectin: does it really matter? Dig Dis Sci. 2012;57:827-829. doi:10.1007/s10620-012-2079-6.

10. Fagerhol MK, Dale I, Andersson T. Release and quantitation of a leukocyte derived protein (L1). Scand J Haematol. 1980;24: 393-398.

11. Sipponen T. Diagnostics and prognostics of inflammatory bowel disease with fecal neutrophil-derived biomarkers calprotectin and lactoferrin. Dig Dis. 2013;31:336-344. doi:10.1159/000354689.

12. Sipponen T, Kolho KL. Fecal calprotectin in diagnosis and clinical assessment of inflammatory bowel disease. Scand J Gastroenterol. 2015;50:74-80. doi:10.3109/00365521.2014.987809.

13. Logan R. Faecal calprotectin for the diagnosis of inflammatory bowel disease. BMJ. 2010;341:c3636. doi:10.1136/bmj.c3636.

14. Røseth AG. Determination of faecal calprotectin, a novel marker of organic gastrointestinal disorders. Dig Liver Dis. 2003;35: 607-609.

15. Sipponen T, Haapamäki J, Savilahti E, et al. Fecal calprotectin and S100A12 have low utility in prediction of small bowel Crohn's disease detected by wireless capsule endoscopy. Scand J Gastroenterol. 2012;47:778-784. doi:10.3109/00365521.2012. 677953.

16. van Rheenen PF, Van de Vijver E, Fidler V. Faecal calprotectin for screening of patients with suspected inflammatory bowel disease: diagnostic meta-analysis. BMJ. 2010;341:c3369. doi:10. 1136/bmj.c3369.

17. Koulaouzidis A, Douglas S, Rogers MA, Arnott ID, Plevris JN. Fecal calprotectin: a selection tool for small bowel capsule endoscopy in suspected IBD with prior negative bi-directional endoscopy. Scand J Gastroenterol. 2011;46:561-566. doi:10. 3109/00365521.2011.551835.

18. Kopylov U, Nemeth A, Koulaouzidis A, et al. Small bowel capsule endoscopy in the management of established Crohn's disease: clinical impact, safety, and correlation with inflammatory biomarkers. Inflamm Bowel Dis. 2015;21:93-100. doi:10. 1097/MIB.0000000000000255.

19. Sliker LJ, Ciuti G. Flexible and capsule endoscopy for screening, diagnosis and treatment. Expert Rev Med Devices. 2014; 11:649-666. doi:10.1586/17434440.2014.941809.

20. Koulaouzidis A, Iakovidis DK, Karargyris A, Plevris JN. Optimizing lesion detection in small-bowel capsule endoscopy: from present problems to future solutions. Expert Rev Gastroenterol Hepatol. 2015;9:217-235. doi:10.1586/17474124.2014.952281.

21. Koulaouzidis A, Giannakou A, Yung DE, Dabos KJ, Plevris JN. Do prokinetics influence the completion rate in small-bowel capsule endoscopy? A systematic review and meta-analysis. Curr Med Res Opin. 2013;29:1171-1185. doi:10.1185/03007995.2013. 818532.

22. Koulaouzidis A, Douglas S, Plevris JN. Blue mode does not offer any benefit over white light when calculating Lewis score in small-bowel capsule endoscopy. World J Gastrointest Endosc. 2012;4:33-37. doi:10.4253/wjge.v4.i2.33.

23. Kopylov U, Seidman EG. Role of capsule endoscopy in inflammatory bowel disease. World J Gastroenterol. 2014;20:11551164. doi:10.3748/wjg.v20.i5.1155.

24. http://www.statstutor.ac.uk/resources/uploaded/spearmans.pdf.

25. Koulaouzidis A, Nemeth A, Johansson GW, Toth E. Dissecting Lewis score under the light of fecal calprotectin; an analysis of correlation of score components with calprotectin levels in capsule endoscopy. Ann Gastroenterol. 2015;28:259-264. 
26. Bolanis P, Stauropoulou E. The ratio of monocytes to CRP correlates to symptoms induction time of the acute phase. 37 Annual Panhellenic Medical congress, May 2011.

27. Sandler RS, Everhart JE, Donowitz M, et al. The burden of selected digestive diseases in the United States. Gastroenterology. 2002;122:1500-1511.

28. Dhaliwal A, Zeino Z, Tomkins C, et al. Utility of faecal calprotectin in inflammatory bowel disease (IBD): what cut-offs should we apply? Frontline Gastroenterol. 2015;6:14-19.

29. Kopylov U, Yablecovitch D, Lahat A, et al. Detection of small bowel mucosal healing and deep remission in patients with known small bowel Crohn's disease using biomarkers, capsule endoscopy and imaging. Am J Gastroenterol. 2015;110: 1316-1323.

30. Olsen PA, Fossmark R, Qvigstad G. Fecal calprotectin in patients with suspected small bowel disease-a selection tool for small bowel capsule endoscopy? Scand J Gastroenterol. 2015;50:272277. doi:10.3109/00365521.2014.1003395.

31. Gisbert JP, McNicholl AG. Questions and answers on the role of faecal calprotectin as a biological marker in inflammatory bowel disease. Dig Liver Dis. 2009;41:56-66.
32. Mao R, Xiao YL, Gao X, et al. Fecal calprotectin in predicting relapse of inflammatory bowel diseases: A meta-analysis of prospective studies. Inflamm Bowel Dis. 2012;18:1894-1899.

33. Summerton CB, Longlands MG, Wiener K, et al. Faecal calprotectin: a marker of inflammation throughout the intestinal tract. Eur J Gastroenterol Hepatol. 2002;14:841-845.

34. Stawczyk-Eder K, Eder P, Lykowska-Szuber L, et al. Is faecal calprotectin equally useful in all Crohn's disease locations? A prospective, comparative study. AMS. 2015;1:353-361.

35. Jensen MD, Kjeldsen J, Nathan T. Fecal calprotectin is equally sensitive in Crohn's disease affecting the small bowel and colon. Scand J Gastroenterol. 2011;46:694-700.

36. Lasson A, Stotzer PO, Öhman L, Isaksson S, Sapnara M, Strid H. The intra-individual variability of faecal calprotectin: a prospective study in patients with active ulcerative colitis. $J$ Crohns Colitis. 2015;9:26-32. doi:10.1016/j.crohns.2014.06.002.

37. Calafat M, Cabré E, Mañosa M, Lobatón T, Marín L, Domènech E. High within-day variability of fecal calprotectin levels in patients with active ulcerative colitis: what is the best timing for stool sampling? Inflamm Bowel Dis. 2015;21:1072-1076. doi:10. 1097/MIB.0000000000000349. 